\title{
FRAME DOMAIN SIGNAL PROCESSING: FRAMEWORK AND APPLICATIONS
}

\author{
Amina Chebira ${ }^{1}$, Matthew Fickus ${ }^{2}$ and Martin Vetterli ${ }^{1}$ \\ ${ }^{1}$ School of Computer and Communication Sciences \\ Ecole Polytechnique Fédérale de Lausanne (EPFL), CH-1015, Switzerland \\ Email: amina.chebira, martin.vetterli@epfl.ch; Web:lcav.epfl.ch/ $\{$ chebira, vetterli $\}$ \\ 2 Dept. of Mathematics and Statistics, Air Force Institute of Technology \\ Wright-Patterson Air Force Base, Ohio 45433, USA \\ Email: matthew.fickus@afit.edu
}

\begin{abstract}
Besides basis expansions, frames representations play a key role in signal processing. We thus consider the problem of frame domain signal processing, which is more complex and challenging than transform domain processing. Examples of such processing abound, from overlap-add/save convolution, to frequency domain LMS, and frame magnitude reconstruction. We develop a unified view of all these situations by using a common Hilbert space view of the problem, and consider algorithms in this common framework. In addition to a synthetic view of multiple signal processing methods in frames, we derive several original results. This include a direct solution to spectral modification (which usually uses an iterative algorithm) and a unicity condition for reconstruction from frame coefficient magnitudes.
\end{abstract}

Index Terms - frames, orthogonal projection, spectral modification, short-time Fourier transform.

\section{INTRODUCTION AND MOTIVATION}

Frames have become one of the basic tools in signal representation and processing [1]. Many standard procedures used in speech processing, adaptive filtering and image processing, to name just a few, can be phrased in frame domain. But unlike orthonormal or biorthogonal basis representations, frame representations are constrained by the range of the frame operator. That is, given an analysis frame $F^{*}$ and $X=F^{*} x$ then $X \in \operatorname{Range}\left(F^{*}\right)$ and any processing of $X$ needs to take this into account. More precisely, if $x \in \mathbb{R}^{N}$ and $S$ the subspace of $\mathbb{R}^{M}(M>N)$ given by $S=\operatorname{Range}\left(F^{*}\right)$, then a change of $X$, or $X=X+\Delta X$, can be decomposed into $\Delta X=\Delta X_{S}+\Delta X_{S \perp}$, of which only $\Delta X_{S}$ will have an effect after reconstruction by a dual frame $F^{\dagger}$, or $x=F^{\dagger} X=F^{\dagger}\left(X+\Delta X_{S}+\right.$ $\left.\Delta X_{S \perp}\right)=x+F^{\dagger} \Delta X_{S}$.

These elementary facts on frame analysis and reconstruction are sometimes hidden inside sophisticated signal processing algorithms. Also, finding $\Delta X_{S}$ closest to a desired modification $\Delta X$ is often performed using an iterative algorithm [2]. In addition, a number of signal processing tasks deal with the magnitude of frame coefficients, which poses both theoretical and algorithmic challenges. For example, is the magnitude representation unique (up to a global sign change) and are there efficient ways to reconstruct $x$ or $X$ given the magnitude of the entries of $X$ ?

The present paper has therefore several goals, from the theory to the practice, but also from the synthetic view to the pedagogical

This work was supported by the Swiss National Science Foundation under grant number 200021-121935. Fickus was supported by AFOSR F1ATA09125G003. exposition. In Section 2, after establishing basic frame facts and notations, we gather various signal processing tasks that are performed in frame domain under a single framework. This ranges from running convolution algorithms (overlap-add/save), to adaptive filtering (frequency-domain LMS), to spectral domain modifications (spectral shaping) and reconstruction from spectrum magnitude. Then, in Section 3, we consider linear modifications and derive an exact operator for a one step solution instead of iterative solutions. This leads to substantial computational savings. In Section 4, we address the problem of frame representations where only the magnitude of the frame coefficients are kept. This is standard for example in spectrograms. This raises the question of uniqueness of such a representation, which is settled with a necessary and sufficient condition. Given this condition being satisfied, we propose an algorithm to reconstruct the unique $X$ from its magnitude. All along, we work both with a very small frame (the archetypal Mercedes-Benz frame) as well as with a medium size short-time Fourier transform (STFT) frame, the first for pedagogical reasons, and the second because of its high relevance in practice.

\section{FRAME SIGNAL PROCESSING}

\subsection{Frame Basics and Definitions}

Frames are redundant sets of spanning vectors in a given Hilbert space $\mathbb{H}$. In this work, we only consider finite dimensional Hilbert spaces $\left(\mathbb{R}^{N}, \mathbb{C}^{N}\right)$. Given a redundant spanning set of vectors $F=$ $\left\{f_{m}\right\}_{m=0}^{M-1}, f_{m} \in \mathbb{C}^{N}, M>N$, we associate to it an $N \times M$ matrix (operator) which we will also call $F$ whose $m$-th column is the frame vector $f_{m}$; this is known as the synthesis frame operator. Let $F^{*}$ be the analysis operator that maps $\mathbb{C}^{N}$ into $\mathbb{C}^{M}$ such that for any $x \in \mathbb{C}^{N}$ and $m=0, \ldots, M-1, X_{m}=\left(F^{*} x\right)_{m}=\left\langle x, f_{m}\right\rangle$ represents the $m$-th frame coefficient of $x$. By definition of a frame, for any $x \in \mathbb{C}^{N}$, the norm of $X$ is bounded from the left and from the right. To reconstruct a signal $x$ from its frame coefficients, we need to use the pseudo-inverse $F^{\dagger}=\left(F F^{*}\right)^{-1} F$ such that $F^{\dagger} F^{*}=I$ (identity matrix) to obtain $x=F^{\dagger} X$.

A particular class of interest are tight frames $(T F S)$ which can be seen as a generalization of orthonormal bases (ONBs). These frames are self-dual such that $F F^{*}=A I$, and norm preserving (up to the constant $A$ ), in which case they are called A-tight. Moreover, if $F$ is a finite dimensional tight frame, then its rows are orthogonal with constant norm. Throughout this paper, $S$ will denote the range of $F^{*}$, $P_{S}=F^{*}\left(F F^{*}\right)^{-1} F$ the -orthogonal- projection onto $S$ and $H^{\perp}$ the orthogonal complement of a subspace $H$. 


\subsection{Examples of Frame Domain Signal Processing}

Many signal processing tasks use frames and process signals in the frame domain. For example, in running convolution algorithms, known as overlap-add/overlap-save [3], the goal is to compute a linear convolution $y=x * g$ using circular convolution,assuming $x, g$ are of finite length $N, L$ respectively, . This is possible when the period of the circular convolution $M$ is such that $M \geq$ $N+L-1$. Assume that DFT is the discrete Fourier transform matrix of size $M, F_{x}^{*}$ the frame consisting of the first $N$ vectors of $D F T$, and $F_{g}^{*}$ the frame consisting of the first vector of DFT and the last $L-1$ vectors of $D F T$ in reverse order. Then, we can compute the result of the linear convolution as $y=D F T^{*} \operatorname{diag}\left(F_{g}^{*} G\right) F_{x}^{*} x$, where $G$ is an $M$-dimensional vector such that $G=\left[g_{0}, 0, \ldots, 0, g_{L-1}, g_{L-2}, \ldots, g_{1}\right]^{\top}$, and $\operatorname{diag}\left(F_{g}^{*} G\right)$ puts the elements of $G$ in a diagonal matrix. Therefore, if we are to modify the elements of $G$, we should do it on Range $\left(F_{g}^{*}\right)$. Likewise, in frequency domain adaptive filtering (LMS), one cannot modify $G$ outside of the range of $F_{g}^{*}$ for risk of obtaining a filter $g$ of full length $(M)$ leading to wrap around effects. Current frequency domain LMS algorithm make use of this fact by either driving the optimization on the range itself [4] (so called "unconstrained" algorithm), or driving the optimization in the $M$-dimensional space then projecting onto the range [5] (so called "constrained" algorithm). Spectrogram modifications $[2,6]$, such as reconstruction from the magnitude of spectrum coefficients, is another set of applications with an underlying frame range. In [2], the authors present two POCS-based algorithms to estimate a signal either from its spectral coefficients or from their magnitude. Balan et. al [7] present fast algorithms for the reconstruction from magnitude of frame coefficients. These are in polynomial time and linear time for specific frames families. They also derive sufficient conditions on the size of the frame to obtain uniqueness of the reconstruction (up to unimodular constants). These consist of $M \geq 2 N-1$ for the real case and $M \geq 4 N-2$ for the complex case.

Within the "analysis-modification-synthesis" framework, modifications in the frame domain can be divided into two main classes, linear and nonlinear ones. In the next section, we study the effect of the former and derive an efficient way to apply linear changes in the spectral domain before reconstruction.

\section{LINEAR MODIFICATIONS IN THE FRAME DOMAIN}

In the problem of linear modifications, we aim at changing the transform coefficients using a linear operator, or, project $F^{*} x$ onto a linear subspace $H$. However, projecting once leads, in general, to a solution that is not consistent, namely $P_{H} F^{*} x \notin S$. For instance, suppose we want to annul some frequency regions in the spectrogram of an excerpt of an audio signal $x$. Then, the frame coefficients in the said region will "raise from the dead". As an example, let us choose a Hanning window of length $N / 2$ with $50 \%$ overlap and 8 frequency bins, then Fig. 1 depicts the original spectrogram for $N=16$ samples of Ravel's Boléro (sampled at $F_{s}=44.1 \mathrm{~K} \mathrm{~Hz}$ ) and its modified spectrogram after annihilating some frequency region, reconstructing and finally analyzing it again using the same STFT. It is clear that the frame coefficients in the specified frequency region are not equal to zero anymore. It is also important to recall that due to the linear dependence of frame vectors, having some frame coefficients be zero will affect the rest of them. In fact, this is true for any type of modification.

Given a signal $x \in \mathbb{C}^{N}$, a frame $F^{*}$ for $\mathbb{C}^{N}$ such that $X=$ $F^{*} x, X \in \mathbb{C}^{M}$ and $M>N$. For example, $F^{*}$ is an STFT frame and $X$ are the corresponding spectral coefficients of $x$. Let $H$ be the

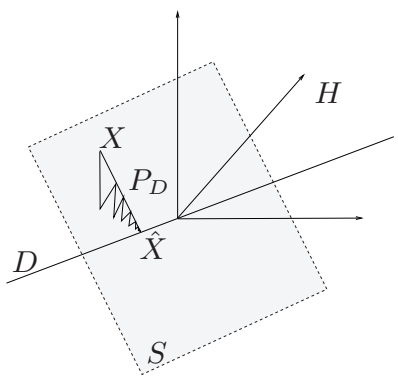

Fig. 2. Example of alternating projections for $N=2, M=3$, where $H$ is the $X Y$-plane, $S$ a given plane and $D=S \cap H$ a line on the $X Y$-plane intersecting $S$.

desired subspace (for example, $H$ is such that $X_{i}=0$ for $i \in \mathcal{I}$, a predefined subset of indices). We would like to find the $\hat{X}$, the closest point to $X$ in $H$, yet, as argued earlier, we also need a consistent solution such that $\hat{X}$ corresponds to an actual signal $\hat{x} \in \mathbb{C}^{N}$, that is, we also want $\hat{X}$ to be in the range of $F^{*}$, called $S$. Therefore, if we let $D=S \cap H$, we formulate our problem as follows: find $\hat{X} \in D$ such that $\hat{X}=\min _{Y \in D}\|X-Y\|$, namely, we want to find $\hat{x}=$ $F^{\dagger} P_{D} F^{*} x$. There are two ways to reach $\hat{X}$ : Either by alternating projections between the range of the analysis frame and the subspace $H$, or via projecting directly onto the intersection $D=S \cap H$.

\subsection{Alternating Projections}

The method of alternating projections is widely used in signal processing applications as it is proven to converge to the optimal point as long as the subspaces are closed convex sets and their intersection is not empty [8]. Specifically, assume $S \cap H \neq \emptyset$ and $X^{(0)}=F^{*} x$ for some $x \in \mathbb{R}^{N}$. Then, the sequence $X^{(k+1)}=P_{S} P_{H} X^{(k)}$ converges in norm to $\hat{X}=P_{D} X^{(0)}$ when $k \rightarrow \infty$; and $\hat{x}=$ $F^{\dagger} \hat{X}$. This algorithm yields the optimal solution but its rate of convergence depends on the principal angles between $S$ and $H$. Indeed, if $\alpha$ is the principal angle between $S$ and $H$ such that $\cos (\alpha)=$ $\sup \left\{\langle X, Y\rangle, X \in S \cap H^{\perp}, Y \in H \cap S^{\perp},\|X\|,\|Y\| \leq 1\right\}$, then Aronszajn [9] proved that for any $X \in \mathbb{R}^{M}$, we have $\|\left(P_{S} P_{H}\right)^{k} X-$ $P_{D} X\left\|\leq \cos (\alpha)^{2 k-1}\right\| X \|$. Namely, the rate of convergence decreases as $\cos (\alpha)^{2 k-1}$. Fig. 2 shows an example of alternating projections for $N=2, M=3$, where $H$ is the $X Y$-plane, $S$ a given plane and $D=S \cap H$ a line on the $X Y$-plane intersecting $S$. We start from a point $X \in S$ then project it onto $H$ and then project onto $S$. We repeat this process until we reach $\hat{X}$ that lies on the line $D$.

In summary, this method not only yields the closest point in $D=$ $S \cap H$ but also converges in norm. However, the dependence of its rate of convergence on the principal angles between the subspaces might lead to an inefficient and very slow algorithm.

\subsection{One-step Projection}

To circumvent the disadvantages of the alternating projections methods, we seek to build the projection $P_{D}$ onto $D$, the intersection of the frame range $S$ with the desired subspace $H$. Using the orthogonal projection onto $D$ ensures that $\hat{X} \in D$ is the closest point to $X \in S$. Let us now derive a closed-form formula for the projection operator on $D$, yielding an efficient one-step projection algorithm to reach the optimal point $\hat{X}$ in the frame domain. Given $H \subseteq \mathbb{C}^{M}$, let $Q: \mathbb{C}^{M} \rightarrow H^{\perp}$ be the projection onto the orthogonal complement of $H$ such that $\operatorname{Range}(Q)=H^{\perp}$. Then, the null space of $Q F^{*}$, denoted by $\mathcal{N}\left(Q F^{*}\right)$, is the set of vectors in $\mathbb{C}^{M}$ whose 


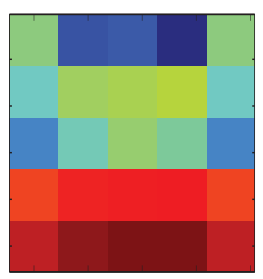

(a)

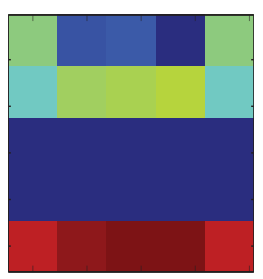

(b)

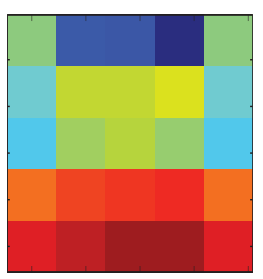

(c)

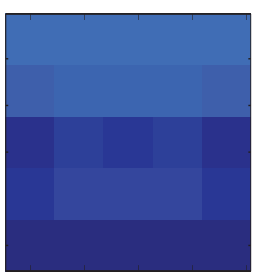

(d)

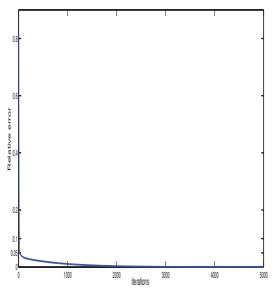

(e)

Fig. 1. Frame coefficients raising from the dead. (a) Original spectrogram, (b) Frequency region annihilated, (c) New spectrogram after one iteration of the alternating projections method, (d) Spectrogram after using the one-step projection, (c) Rate of convergence of the alternating method projection.

frame coefficients are orthogonal to the $\operatorname{Range}(Q)=H^{\perp}$, therefore, $\mathcal{N}\left(Q F^{*}\right)=\left\{x \in \mathbb{C}^{N}, Q F^{*} x \in H\right\}$. Hence, our problem consists of finding a basis (in $\left.\mathbb{C}^{N}\right)$ for $\mathcal{N}\left(Q F^{*}\right)$, or equivalently, build the projection $P$ onto $\mathcal{N}\left(Q F^{*}\right)$.

For instance, suppose that we want to set a number of frame coefficients to zero before we reconstruct the signal (this could be thought of as some denoising process), and assume that $\mathcal{I} \subset\{1, \ldots, M\}$ is the subset of indices for which $\hat{X}_{i}=0, i \in \mathcal{I}$ and let $\mathcal{I}$ have cardinality $|\mathcal{I}|$ (dimension of $H^{\perp}$ ). Note that in general, it is expected that $\hat{X}_{i} \neq X_{i}$, for $i \notin \mathcal{I}$, due to the linear dependence of the frame vectors. Let $Q$ be the operator on $\mathbb{C}^{M}$ such that $(Q X)_{i}=0$ for $X=F^{*} x$ and $i \in \mathcal{I}$. From a different perspective, this problem amounts to looking for $\hat{x} \in \mathbb{C}^{N}$ such that $\left\langle\hat{x}, f_{i}\right\rangle=0$ for $i \in \mathcal{I}$, that is, we would like to find $\hat{x} \in\left(\operatorname{span}\left\{f_{i}\right\}_{i \in \mathcal{I}}\right)^{\perp}$. Using $\mathcal{N}\left(Q F^{*}\right)=\operatorname{Range}\left(F Q^{*}\right)^{\perp}$, one can then easily check that in this particular case, we indeed have $\mathcal{N}\left(Q F^{*}\right)=\left(\operatorname{span}\left\{f_{i}\right\}_{i \in \mathcal{I}}\right)^{\perp}$. Returning to the more general set up, the projection $P$ onto $\mathcal{N}\left(Q F^{*}\right)$ can be written as

$$
P=I-F Q^{*}\left(Q F^{*} F Q^{*}\right)^{-1} Q F^{*},
$$

where $I$ is the identity matrix in $\mathbb{R}^{N}$. To have a stable projection, it is necessary that $K \leq N$ (otherwise, we can only reconstruct the zero sequence). In fact, to write $P$ as in (1), we need the columns of $Q F^{*}$ to be linearly independent. Using a Gram-Schmidt procedure to transform this spanning set for $\mathcal{N}\left(Q F^{*}\right) \subseteq \mathbb{C}^{N}$ into an ONB solves this issue. Note that when we want some spectral coefficients to be zero, then this is equivalent to find a basis for the set $\left\{f_{i}\right\}_{i \in \mathcal{I}}$.

Given that $P$ is a projection onto a subspace of $\mathbb{C}^{N}$, we should use $F^{*}$ to return to the frame domain and find the projection onto $D$. Namely, $D=\operatorname{Range}\left(F^{*} P\right)$.

Proposition 1 Suppose $G$ is a matrix whose columns are the basis vectors of $\mathcal{N}\left(Q F^{*}\right)$. Then, for any frame $F$ of size $M$ in $\mathbb{R}^{N}(M>$ $N$ ), the projection onto $D$ is

$$
P_{D}=F^{*} G\left(G^{*} F F^{*} G\right)^{-1} G^{*} F
$$

We can easily check that $P_{D}$ is indeed an orthogonal projection, as well as $P_{S} P_{D}=P_{D}$ and $P_{D} Q^{*}=0$.

Now, assume that $F$ is an $A$-tight frame, then $P_{D}=\frac{1}{A} F^{*} P F$. Using (1), this leads to

Proposition 2 If $F$ is an A-tight frame for $\mathbb{R}^{N}$, then

$$
P_{D}=\frac{1}{A} F^{*} F\left(I-Q^{*}\left(Q F^{*} F Q^{*}\right)^{-1} Q F^{*} F\right) .
$$

In particular, if for any $X \in \mathbb{C}^{M}$, we let $\hat{x}=\frac{1}{A} F P_{D} X$, then $\hat{x}$ can be computed as the projection $P$ onto $\mathcal{N}\left(Q F^{*}\right)$ of the reconstruction of $x$ (from $X)$, namely

$$
\hat{x}=\frac{1}{A} P F X .
$$

When $X=F^{*} x$ in the above, then $\hat{x}=P x$. Moreover, note that when $G$ is an ONB, $P_{D}=\frac{1}{A}\left(F^{*} G\right)\left(F^{*} G\right)^{*}$, that is, $P_{D}$ is the frame operator of $F^{*} G$ (up to a scalar).

Example 1 a. Mercedez-Benz frame. Let

$$
F_{m b}^{*}=\left[\begin{array}{cc}
0 & \sqrt{\frac{2}{3}} \\
\frac{-1}{\sqrt{2}} & \frac{-1}{\sqrt{6}} \\
\frac{1}{\sqrt{2}} & \frac{-1}{\sqrt{6}}
\end{array}\right], \quad \text { then } \quad P_{S}=\frac{1}{3}\left[\begin{array}{ccc}
2 & -1 & -1 \\
-1 & 2 & -1 \\
-1 & -1 & 2
\end{array}\right]
$$

This is the Mercedes-Benz frame for $\mathbb{R}^{2}$. It is A-tight with $A=1$ and its range $S$ consists of the plane with normal $[1,1,1]^{\top}$. Let $X=F_{m b}^{*} x$ for some $x \in \mathbb{R}^{2}$ and suppose we want to find $y=\left[y_{0}, y_{1}\right]^{\top}$ such that $F^{*} y=Y=\left[Y_{1}, Y_{2}, 0\right]^{\top}$ where $Y_{1}, Y_{2}$ are as close as possible to $X_{1}, X_{2}$, respectively. This implies that $H$ consists of the $X Y$-plane. Using the alternating projections methods leads to computing $X^{(k+1)}=P_{S} P_{H} X^{(k)}$ for increasing values of $k$. Alternatively, we can directly compute the projection onto the intersection of $S$ and $H$ (see Fig. 2), or as we have seen, compute the projection onto the orthogonal complement to $\operatorname{span}\left\{f_{3}\right\}$ with $\left[\frac{1}{\sqrt{2}}, \frac{-1}{\sqrt{6}}\right]^{\top}$. That is compute $P=I-P_{\left\{f_{3}\right\}}$. For $x=[-6,-3]^{\top}$, we find

$$
P=\left[\begin{array}{cc}
\frac{1}{4} & \frac{\sqrt{3}}{4} \\
\frac{\sqrt{3}}{4} & \frac{3}{4}
\end{array}\right] \text { and } \hat{x}=\left[\begin{array}{c}
-\frac{6-3 \sqrt{3}}{4} \\
\frac{-6 \sqrt{3}-9}{4}
\end{array}\right],
$$

which is the same as with the alternating projections method (which converges after $k=10$ iterations for the chosen $x$ ).

b. STFT. Now let us consider the spectrogram shown in Fig. 1(a) and described earlier in Section 3. For a signal length $N=16$, we compute 40 STFT coefficients using $L=8$ frequency bins, a Hanning window $g$ of length $L_{g}=\frac{N}{2}$ and $50 \%$ overlap. Let $\delta=\frac{L_{g}}{2}$, then the frame coefficients of a signal $x$ are $X(k, l)=\langle x, g(k, l)\rangle$ where $g(k, l)=g_{n-k \delta} e^{-j 2 \pi l / L}$ for $k=-1, \ldots, 3, l=0, \ldots L-1$. We consider annihilating the coefficients $X(k, l)$ for $l=1,2$ and all $k$ (see Fig. 1(b)), therefore, using the one-step projection method leads to computing the projection onto the span of $\{g(k, 1), g(k, 2)\}_{k=-1}^{3}$. The result is shown on Fig. 1(d). Fig. 1(e) depicts the rate of convergence of the alternating projections method as a function of the number of iterations. 


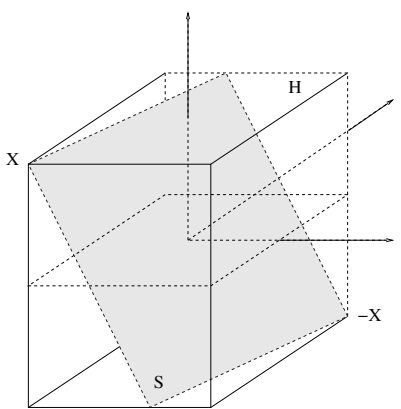

Fig. 3. Reconstruction from magnitude for $N=2, M=3$. The frame range $S$ and the hyperrectangle $H$ formed out of the 8 vertices $Y^{(l)}=B_{l} X . S$ intersects $H$ at $\pm X$ and at two other points on the edges of $H$

\section{RECONSTRUCTION FROM MAGNITUDES OF FRAME COEFFICIENTS}

Assume we have a real frame $F^{*}$ such that $X=F^{*} x$ with $x \in$ $\mathbb{R}^{N}, X \in \mathbb{R}^{M}$ and let us consider the magnitude vector $Y$ of the frame expansion coefficients such that $Y_{m}=\left|X_{m}\right|$. We ask the following question: When does $Y$ uniquely determine $X$ (up to $\pm X$ ) and thus $x$ ? Given $Y$, there is a set of $2^{M}$ possible vectors $Y^{(l)}, l=$ $0, \ldots, 2^{M}-1$ that share the same $Y$, amongst them, there is $X$ and $-X$. As $X \in S$, we can test all $Y^{(l)}$ for membership in $S$. Consider the following matrices that act on the set of frame coefficients to change their sign:

$$
B_{l}=\operatorname{diag}\left[\begin{array}{llll}
(-1)^{i_{0}} & (-1)^{i_{1}} & \ldots & (-1)^{i_{M-1}}
\end{array}\right],
$$

where $i_{m}$ is the $m$-th bit of the binary expansion of $l$. Then, we have $Y^{(l)}=B_{l} Y$. Therefore, given $Y$, we have to search for $\widehat{Y}^{(l)}$ such that $\widehat{Y}^{(l)}=\widehat{B}_{l} Y \in S$ and if it is unique (up to \pm 1 ), then we have found the unique solution. We need to prove that except for $X$ and $-X$, there is no other $Y^{(l)}$ satisfying $Y^{(l)}=P_{S} Y^{(l)}$. Thus, given that $P_{S}$ is a projection, $X=B_{0} X$ and $-X=B_{2^{M-1}} X$, uniqueness is violated whenever there exists $l \in\left\{1, \ldots, 2^{M}-2\right\}$ such that $B_{l} P_{S} B_{l}=P_{S}$. This test can be conducted with all $2^{M}-2$ matrices $B_{l}$.

An equivalent argument that is rather geometrical is as follows: Let $\widetilde{H}=\left\{Y^{(l)}\right\}_{l=0}^{2^{M}-1}$. That is, $\widetilde{H}$ is the set of the summits of the hyperrectangle $H$ of dimension $M$, with vertices $\left\{ \pm X_{0}, \pm X_{1}, \ldots\right.$, $\left.\pm X_{M-1}\right\}$. To prove uniqueness of the reconstruction (up to \pm 1 ), we need to show that the only vertices on $S$ are $\pm X$. Figure 3 shows schematically $H$ and $S$ for $N=2, M=3$. We observe that the intersection of $S$ and $H$ contains only $2^{N}$ points, two of which are the vertices $\pm X$. If we construct the $M \times 2^{M}$ matrix $\Phi$ whose columns are the $2^{M}-1$ vectors $\varphi_{l}=\left[i_{0}, i_{1}, \ldots, i_{M-1}\right]^{\top}$ for $l=1, \ldots, 2^{M}-1$, then, we need to verify that none of the $\varphi_{l}$ 's is preserved through the operator $P_{S} \Phi$. Note that $\varphi_{0}, \varphi_{2^{M}-1}$ correspond to $X,-X$, respectively.

Proposition 3 For any real analysis frame $F^{*}$ and the projection $P_{S}$ onto its range, the reconstruction from the magnitudes of the frame expansion coefficients is unique (up to \pm 1 ) if and only if $P_{S} \Phi$ does not preserve any of the columns $\varphi_{l}$ of $\Phi$ for $l=1, \ldots, 2^{M}-2$.

The above is indeed a necessary condition for uniqueness, because if violated, then there exists a vertex in $\widetilde{H} \cap S$ that is different from $\pm X$. For instance, let us choose $M=3, N=2$ and

$$
F^{*}=\left[\begin{array}{cc}
1 & 0 \\
0 & \frac{1}{\sqrt{2}} \\
0 & \frac{1}{\sqrt{2}}
\end{array}\right], \quad \text { then } \quad P_{S}=\left[\begin{array}{ccc}
1 & 0 & 0 \\
0 & \frac{1}{2} & \frac{1}{2} \\
0 & \frac{1}{2} & \frac{1}{2}
\end{array}\right] \text {, }
$$

and $P_{S} \varphi_{1}=\varphi_{1}=[1,0,0]^{\top}$ as well as $P_{S} \varphi_{5}=\varphi_{5}=[0,1,1]^{\top}$. Thus, along with $\pm X$, we have two additional vertices that belong to $\widetilde{H} \cap S$, invalidating the uniqueness of the reconstruction. Note that whenever $Y^{(l)} \in \widetilde{H} \cap S$, then the same is true for $-Y^{(l)}$. From this example, we see that whenever $S$ is aligned with one of the faces of the hyperrectangle $H$ or contains a subset of vectors from the canonical ONB in $\mathbb{R}^{M}$ then the reconstruction is not unique.

It is also a sufficient condition. Given that $\Phi$ covers all the vertices of $H$ and if for all $l=1, \ldots, 2^{M}-2$, none of the $\varphi_{l}$ is preserved through $P_{S}$, then $P_{S} Y^{(l)} \neq Y^{(l)}$ for all $l=1, \ldots, 2^{M}-2$. Thus, the feasible set $S \cap \widetilde{H}$ contains only $\pm X$, which concludes the argument.

We again observe here the essential role played by $P_{S}$. In addition, this test provides a necessary and sufficient condition that can also be used to drive the design of $P_{S}$ or the frame itself to ensure uniqueness of reconstruction from the magnitudes of the frame expansion coefficients.

Let us now consider an algorithm of reconstruction. One of the possible methods is through quadratic programming, whereby we do the following: find $\hat{x}$ that maximizes $\|\hat{x}\|$ under the constraints $\left[\begin{array}{c}F^{*} \\ -F^{*}\end{array}\right] \hat{x} \leq\left[\begin{array}{c}Y \\ Y\end{array}\right]$. The feasible set for this program is a polygon of at most $2^{N}$ vertices that consists of the intersection of $S$ with the convex hull of $H$.

\section{CONCLUSIONS}

This work is a first step towards creating a unifying framework for frame domain signal processing. Specifically, we have derived a onestep procedure to perform linear modifications in the frame domain, leading to an efficient algorithm that can be used as an alternative to methods based on iterative projections onto convex sets. We have also established a necessary and sufficient condition for reconstruction from the magnitude of real frame coefficients.

\section{References}

[1] J. Kovačević and A. Chebira, "Life beyond bases: The advent of frames (Part I)," IEEE Signal Proc. Mag., vol. 24, no. 4, pp. 86-104, Jul. 2007.

[2] D. Griffin and J. Lim, "Signal estimation from modified shorttime Fourier transform," IEEE Trans. Acoust., Speech, and Signal Proc., vol. 32, no. 2, pp. 236-243, 1984.

[3] A. V. Oppenheim, R. W . Schafer, and I. T. Young, Digital Signal Processing, Prentice Hall, Englewood Cliffs, NJ, 1975.

[4] A. Feuer and R. Cristi, "On the steady state performance of frequency domain LMS algorithm," IEEE Trans. Signal Proc., vol. 41, no. 1, pp. 419-423, 1993.

[5] J. J. Shynk, "Frequency domain and multirate adaptive filtering," IEEE Signal Proc. Mag., vol. 9, no. 1, pp. 14-37, 1992.

[6] J. B. Allen and L. R. Rabiner, "A unified approach to short-time Fourier analysis and synthesis," Proc. IEEE, vol. 65, no. 11, pp. 1558-1564, 1977.

[7] R.Balan, B.G.Bodmann, P.G.Casazza, and D.Edidin, "Fast algorithms for signal reconstruction without phase," vol. 6701, pp. 67011L, Aug. 2007.

[8] J. Von Neumann, Functional Operators: The Geometry of Orthogonal Spaces, Volume 2, Princeton University Press, 1950.

[9] N. Aronszajn, "Theory of reproducing kernels," Trans. Amer. Math. Soc., vol. 68, pp. 337-404, 1950. 